\title{
Oct $3 / 4$ is potentially useful for the suppression of the proliferation and motility of hepatocellular carcinoma cells
}

\author{
MINORU TOMIZAWA ${ }^{1}$, FUMINOBU SHINOZAKI ${ }^{2}$, YASUFUMI MOTOYOSHI ${ }^{3}$, \\ TAKAO SUGIYAMA $^{4}$, SHIGENORI YAMAMOTO ${ }^{5}$ and NAOKI ISHIGE ${ }^{6}$ \\ Departments of ${ }^{1}$ Gastroenterology, ${ }^{2}$ Radiology, ${ }^{3}$ Neurology, ${ }^{4}$ Rheumatology, ${ }^{5}$ Pediatrics and ${ }^{6}$ Neurosurgery, \\ National Hospital Organization, Shimoshizu Hospital, Yotsukaido, Chiba 284-0003, Japan
}

Received May 4, 2016; Accepted February 27, 2017

DOI: $10.3892 / 01.2018 .9292$

\begin{abstract}
Hepatocellular carcinoma (HCC) cells are immature compared with healthy mature hepatocytes. Transcription factors serve a role in hepatocyte differentiation. The expression levels of transcription factors in HCC cell lines have been investigated to determine potential therapeutic targets. In the present study, the HLE, HLF, PLC/PRF/5, Huh-7, Hep3B, Huh-6 and HepG2 HCC cell lines were subjected to reverse-transcription polymerase chain reaction (RT-PCR) of transcription factors, including NANOG, Oct3/4, GATA binding protein 4 (GATA4), GATA6 and hematopoietically expressed homeobox (HHEX). In addition, these cell lines were analyzed using RT-quantitative PCR (RT-qPCR) of NANOG and Oct3/4. The 201B7 human induced pluripotent stem cells were evaluated as a model of pluripotent cells. The HLF cells were transfected with Oct3/4 small interfering RNA (siRNA) and used in an MTS colorimetric assay and a scratch assay. NANOG was not expressed in any of the cell lines. However, GATA4, GATA6 and HHEX were expressed in the majority of the HCC cell lines. In addition, NANOG and Oct3/4 were expressed in 201B7 cells. Oct3/4 was expressed in HLE, HLF and Hep3B cells; however, its expression levels were significantly reduced compared with those in 201B7 cells. RT-qPCR demonstrated that the expression of Oct3/4 siRNA suppressed the proliferation and motility of HLF cells. Oct $3 / 4$ siRNA may be a potentially effective therapy for the suppression of the proliferation and motility of HCC cells.
\end{abstract}

\section{Introduction}

Hepatocellular carcinoma (HCC) develops in the liver following long-term infection with the hepatitis $\mathrm{B}$ or $\mathrm{C}$ virus (1).

Correspondence to: Dr Minoru Tomizawa, Department of Gastroenterology, National Hospital Organization, Shimoshizu Hospital, 934-5 Shikawatashi, Yotsukaido, Chiba 284-0003, Japan E-mail: nihminor-cib@umin.ac.jp

Key words: Oct3/4, small interfering RNA, MTS assay, scratch assay, hepatocellular carcinoma
One of the biological characteristics of HCC is that the cancer cells are similar to immature hepatocytes (2). Additionally, HCC cells are positive for cluster of differentiation (CD)133, a cancer stem cell marker (3), and for biliary epithelial cell markers (3). These results suggest that HCC cells have the potential to differentiate into hepatocytes and biliary epithelial cells (4).

Transcription factors serve an important role in hepatocyte differentiation. For example, CCAAT/enhancer-binding protein $(\mathrm{C} / \mathrm{EBP}) \alpha$ promotes the maturation of immature hepatocytes (5), but it is downregulated in HCC (6). Similarly, GATA binding protein (GATA) 4 and GATA6 are involved in hepatocyte differentiation. Whilst GATA4 is associated with endoderm differentiation (7), GATA6 is required for liver bud formation (8). In addition, hematopoietically-expressed homeobox (HHEX) is essential for the differentiation of human induced pluripotent stem (iPS) cells into hepatocytes (9). These studies indicate that GATA4, GATA6 and HHEX may be involved in the carcinogenesis of HCC.

iPS cells are generated from adult cells upon the introduction of reprogramming factors, including NANOG, sex determining region $\mathrm{Y}$ box 2 (Sox2), Oct3/4 and Krüppel-like factor (KLF)4 (10). The expression patterns of these genes in HCC cells have yet to be elucidated. Therefore, the present study investigated the expression patterns of these transcription factors in order to identify potential novel treatments for HCC. Human iPS cells were used as a model for pluripotent stem cells to compare the expression patterns of these genes.

\section{Materials and methods}

Cell culture. The 201B7 iPS cells (RIKEN BioResource Center, Tsukuba, Japan) were cultured in ReproFF (ReproCELL, Yokohama, Japan) in 6-well plates (Asahi Techno Glass Corporation, Funabashi, Japan) coated with Matrigel ${ }^{\mathrm{TM}}$ (BD Biosciences, Franklin Lakes, NJ, USA), in feeder-cell-free conditions at $37^{\circ} \mathrm{C}$ in an incubator with $5 \% \mathrm{CO}_{2}$. ReproFF was a complete medium and ready to use. The cells were harvested with Accutase ${ }^{\circledR}$ (Innovative Cell Technologies, Inc., San Diego, CA, USA) and seeded onto 6-well plates or 96-well plates coated with Matrigel ${ }^{\mathrm{TM}}$ at a density of $10^{6}$ cells/well. The HLE, HLF, PLC/PRF/5, Huh-7, Hep3B, Huh-6 and HepG2 HCC cell lines were also purchased from RIKEN BioResource Center. 
The HCC cells were cultured in Dulbecco's modified Eagle's medium (DMEM), supplemented with 10\% fetal bovine serum (FBS; Thermo Fisher Scientific, Inc., Waltham, MA, USA) at $37^{\circ} \mathrm{C}$ in an incubator with $5 \% \mathrm{CO}_{2}$. The $\mathrm{HCC}$ cells were trypsinized, harvested and plated onto 6-well plates or 96-well plates. The cells were observed under a light microscope (CKX41N-31PHP; Olympus Corporation, Tokyo, Japan).

Transfection and cell proliferation assay. The HLF cells were trypsinized, harvested and plated onto 96-well flat-bottom plates (Asahi Techno Glass Corporation) at a density of 1,000 cells/well, and then incubated for $24 \mathrm{~h}$ in DMEM supplemented with $10 \% \mathrm{FBS}$ at $37^{\circ} \mathrm{C}$ in an incubator with 5\% $\mathrm{CO}_{2}$. The cells were transfected with negative control small interfering RNA (siRNA) of random sequences or with Oct3/4 siRNA at 20 or $200 \mathrm{nM}$ using Lipofectamine ${ }^{\circledR} 2000$ (all from Thermo Fisher Scientific, Inc.) according to the manufacturer's protocol, and cultured in DMEM supplemented with $10 \%$ FBS at $37^{\circ} \mathrm{C}$ in $5 \% \mathrm{CO}_{2}$. The sequence of the Oct $3 / 4$ siRNA was 5'-CACCCUUUGUGUUCCCAAUUCCUUC-3'. The negative control siRNA was Stealth RNAi ${ }^{\mathrm{TM}}$ siRNA Negative Control, MedGC (cat. no. 12935-300; Thermo Fisher Scientific, Inc). Transfection with Lipofectamine ${ }^{\circledR} 2000$ and no nucleic acid material was used as a mock transfection control. Furthermore, the siRNA-treated cells were cultured for $72 \mathrm{~h}$ and an MTS colorimetric assay was performed according to the manufacturer's protocol (CellTiter $966^{\circledR}$ Aqueous One Solution Cell Proliferation Assay; Promega Corporation, Madison, WI, USA). MTS was ready to use (11). Cells reduce MTS into formazan, a colored product, which has a maximum absorbance at a wavelength of $490 \mathrm{~nm}$; this was measured using an iMark Microplate Absorbance reader (Bio-Rad Laboratories, Inc., Hercules, CA, USA).

Scratch assay. HLF cells were plated onto 4-well chamber slides (BD Biosciences) at a density of $5 \times 10^{4}$ cells/well. When the cells were confluent, the cell sheets were scratched with a sterile razor. The cells were immediately transfected with Oct $3 / 4$ siRNA or the mock transfection control as aforementioned. After 2 days of culture, the cells were stained with hematoxylin and eosin, observed using an AX80 light microscope (magnification, x100; Olympus Corporation) and five distinct fields were imaged; the five distinct fields were chosen randomly. The distance between the edge of the cell sheets and the scratched line was measured. The experiment was repeated three times.

Reverse transcription polymerase chain reaction (RT-PCR) and $R T$-quantitative PCR $(R T-q P C R)$. Total RNA $(5 \mu \mathrm{g})$ was isolated using Isogen ${ }^{\circledR}$ (Nippon Gene Co., Ltd., Tokyo, Japan) from cells cultured in 6-well plates, was utilized for first-strand complementary (c)DNA synthesis, using SuperScript ${ }^{\circledR}$ III First Strand Synthesis and oligo(dT) and following the manufacturer's protocol (Thermo Fisher Scientific, Inc.). RNA from human fetal and adult liver was purchased from Clontech Laboratories, Inc. (Mountain View, CA, USA). PCR was performed using Taq DNA polymerase (Thermo Fisher Scientific, Inc.) and products were separated using gel electrophoresis in $2 \%$ agarose in $1 \mathrm{X}$ TAE $(40 \mathrm{mM}$ Tris-acetate $/ 1 \mathrm{mM}$ EDTA). PCR primer (Thermo Fisher Scientific,Inc.) sequences,

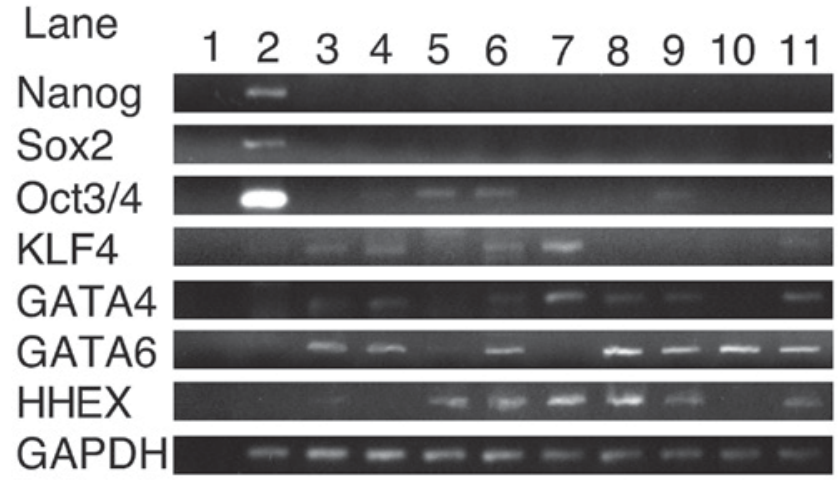

Figure 1. Expression levels of NANOG, Sox2, Oct3/4, KLF4, GATA4, GATA6 and HHEX in control and HCC cell lines as measured by reverse transcription polymerase chain reaction. Lane $1, \mathrm{H}_{2} \mathrm{O} ; 2,201 \mathrm{~B} 7 ; 3$, fetal liver; 4, adult liver; 5, HLE; 6, HLF; 7, PLC/PRF/5; 8, Huh-7; 9, Hep3B; 10, Huh-6 and 11, HepG2 cells. HCC, hepatocellular carcinoma; Sox 2, sex determining region Y box 2; KLF4, Krüppel-like factor 4; GATA, GATA binding protein; HHEX, hematopoietically expressed homeobox.

annealing temperatures, reaction cycle numbers and amplicon lengths for RT-PCR are presented in Table I. RT-qPCR was performed using the Fast SYBR-Green Master Mix (Thermo Fisher Scientific, Inc.) and analyzed with the MiniOpticon ${ }^{\mathrm{TM}}$ Detection System (Bio-Rad Laboratories, Inc.). RT-qPCR was performed for 40 cycles of two steps consisting of $5 \mathrm{sec}$ denaturation at $95^{\circ} \mathrm{C}$ and $5 \mathrm{sec}$ annealing-extension at $60^{\circ} \mathrm{C}$. PCR primers (Thermo Fisher Scientific, Inc.), annealing temperatures, reaction cycle numbers and amplicon lengths for RT-qPCR are listed in Table II. GAPDH and ribosomal protein L19 (RPL19) were used as internal controls for RT-PCR and RT-qPCR, respectively. RPL19 was used as an endogenous control to monitor the quantity of mRNA as a constitutively expressed housekeeping gene (12). The gene expression levels were analyzed using the automated MiniOpticon ${ }^{\mathrm{TM}}$ system based on the $\Delta \Delta$ cycle threshold ( $\Delta \Delta \mathrm{Cq}$ ) method (13). The relative expression was calculated as the expression level of a specific gene divided by the expression level of RPL19. The experiments were performed three times, and triplicates were used in each experiment.

Statistical analysis. One-way analysis of variance was applied for statistical analysis. JMP 10.0.2 software (SAS Institute Inc., Cary NC, USA) was used for statistical analysis. $\mathrm{P}<0.05$ was considered to indicate a statistically significant result.

\section{Results}

RT-PCR indicates the expression levels of transcription factors in HCC cells. To reveal the expression patterns of transcription factors in HCC cells, RT-PCR was performed (Fig. 1). Total RNA from fetal and adult liver cells was analyzed to search for genes expressed in HCC cells but not in fetal and adult liver. These genes may be involved in proliferation of HCC. Whilst NANOG and Sox 2 were not expressed in any of the HCC cell lines, Oct3/4 was expressed in HLE, HLF and Hep3B cells (Lanes 5, 6 and 9, respectively), and GATA4, GATA6 was expressed in HLF, Huh-7, Hep3B, Huh-6 and HepG2 cells. HHEX was expressed in HLE, HLF, PLC/PRF/5, Hep3B, Huh-7 and HepG2 cells. KLF4 was expressed in fetal and 


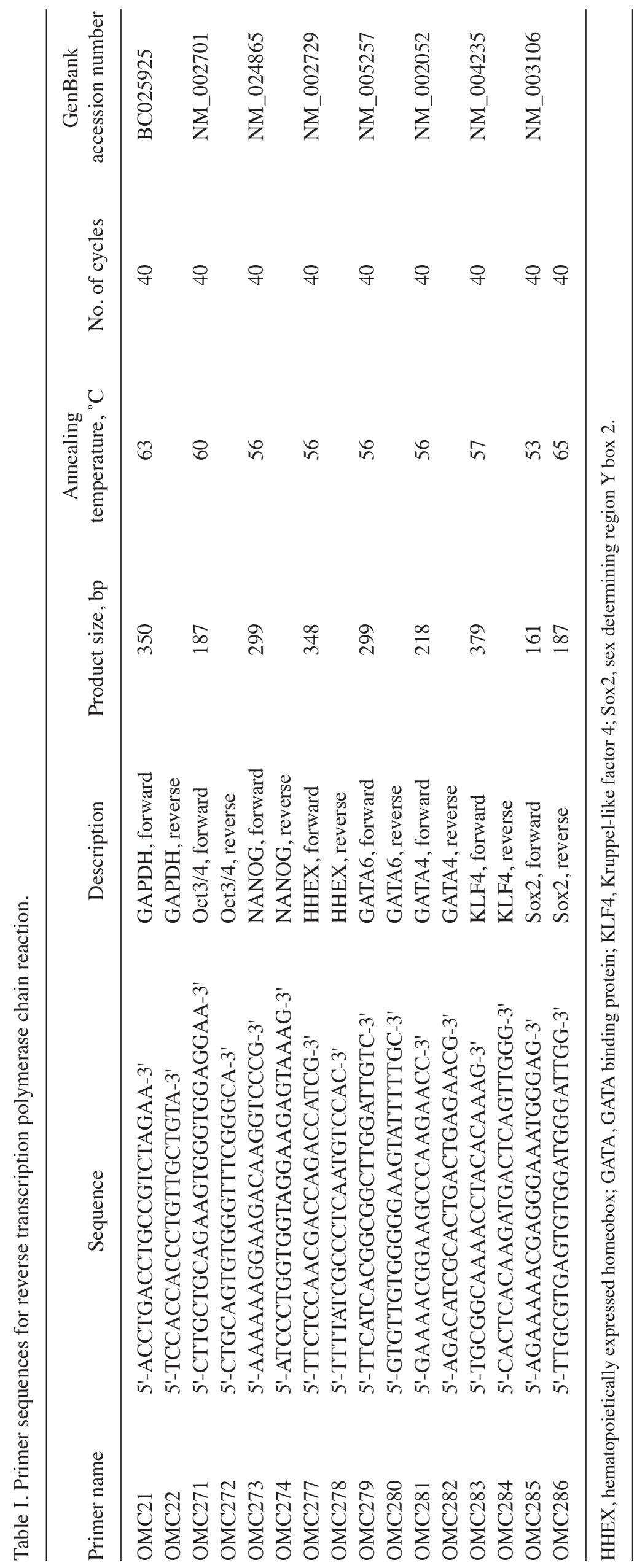



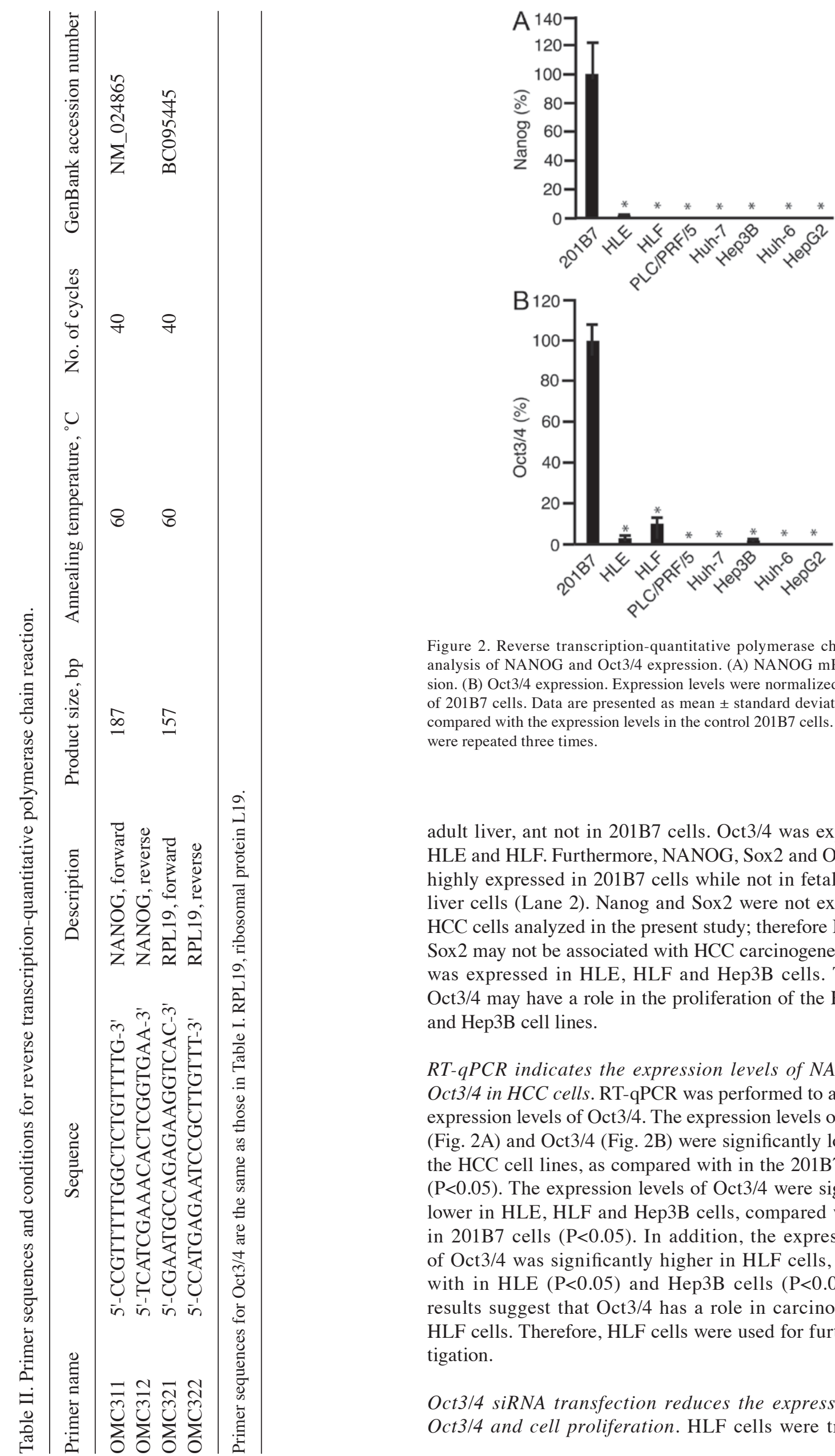

Figure 2. Reverse transcription-quantitative polymerase chain reaction analysis of NANOG and Oct3/4 expression. (A) NANOG mRNA expression. (B) Oct3/4 expression. Expression levels were normalized against that of $201 \mathrm{~B} 7$ cells. Data are presented as mean \pm standard deviation, ${ }^{*} \mathrm{P}<0.05$, compared with the expression levels in the control 201B7 cells. Experiments were repeated three times.

adult liver, ant not in 201B7 cells. Oct3/4 was expressed in HLE and HLF. Furthermore, NANOG, Sox 2 and Oct3/4 were highly expressed in 201B7 cells while not in fetal and adult liver cells (Lane 2). Nanog and Sox2 were not expressed in HCC cells analyzed in the present study; therefore Nanog and Sox 2 may not be associated with HCC carcinogenesis. Oct3/4 was expressed in HLE, HLF and Hep3B cells. Therefore, Oct $3 / 4$ may have a role in the proliferation of the HLE, HLF and Hep3B cell lines.

RT-qPCR indicates the expression levels of NANOG and Oct $3 / 4$ in HCC cells. RT-qPCR was performed to analyze the expression levels of Oct $3 / 4$. The expression levels of NANOG (Fig. 2A) and Oct3/4 (Fig. 2B) were significantly lower in all the HCC cell lines, as compared with in the $201 \mathrm{~B} 7 \mathrm{iPS}$ cells $(\mathrm{P}<0.05)$. The expression levels of Oct $3 / 4$ were significantly lower in HLE, HLF and Hep3B cells, compared with those in $201 \mathrm{~B} 7$ cells $(\mathrm{P}<0.05)$. In addition, the expression level of Oct $3 / 4$ was significantly higher in HLF cells, compared with in HLE $(\mathrm{P}<0.05)$ and Hep3B cells $(\mathrm{P}<0.05)$. These results suggest that Oct $3 / 4$ has a role in carcinogenesis in HLF cells. Therefore, HLF cells were used for further investigation.

Oct3/4 siRNA transfection reduces the expression levels Oct $3 / 4$ and cell proliferation. HLF cells were transfected 

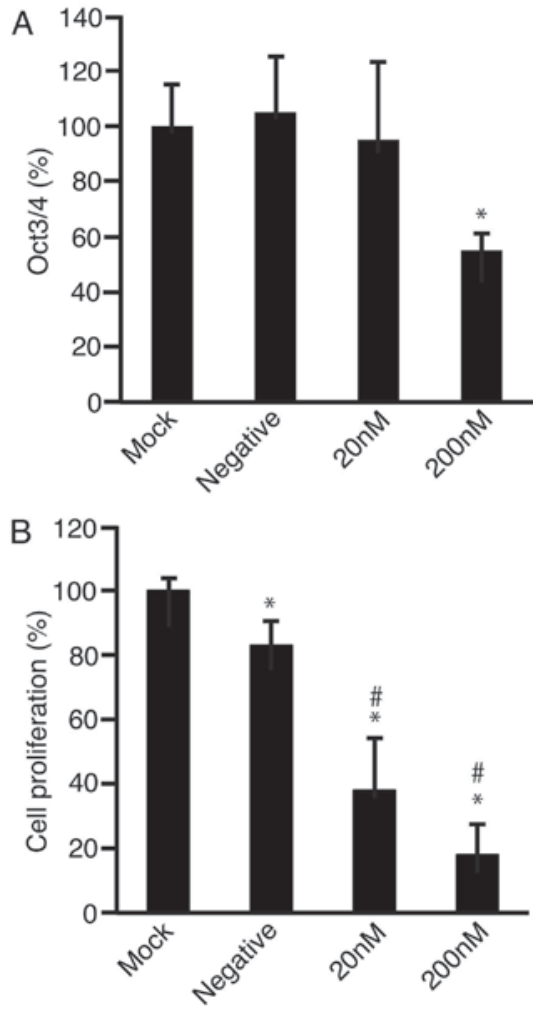

Figure 3. HLF cell proliferation significantly decreases following transfection with Oct3/4 siRNA. Oct3/4 mRNA expression levels in untreated (mock) HLF cells or HLF cells transfected with negative control or Oct3/4 siRNA. (B) HLF cell proliferation following transfection. After 3 days of culturing, the transfected cells were subjected to an MTS colorimetric assay. Data are presented as mean \pm standard deviation, ${ }^{*} \mathrm{P}<0.05$, compared with the mock transfection control, ${ }^{~} \mathrm{P}<0.01$, compared with negative control. Experiments were repeated three times.

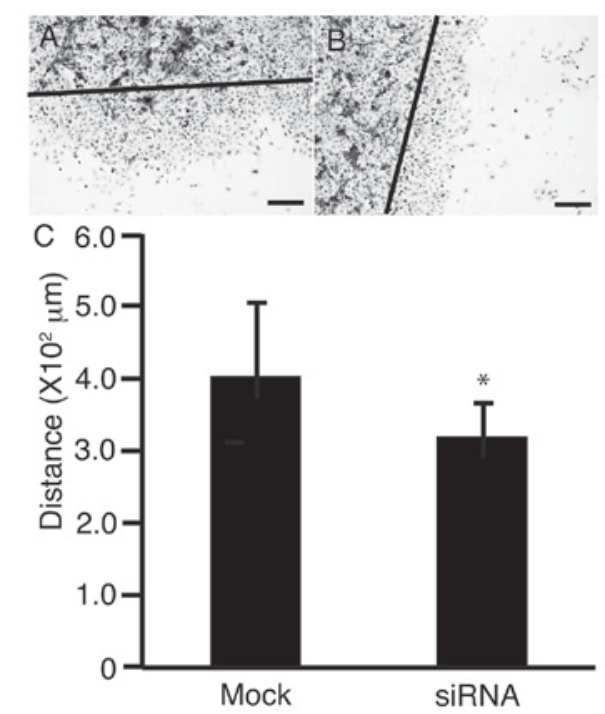

Figure 4 . Oct $3 / 4$ siRNA significantly inhibits the ability of HLF cells to migrate. Scratch wound assay for (A) untreated (mock) HLF cells and (B) HLF cells treated with Oct3/4 siRNA. The cells were stained with hematoxylin and eosin. Distances from the edge of the cell sheet to the scratched line (solid line) were measured at five individual points. (C) Quantification of the distance migrated by untreated and Oct3/4 siRNA-treated HLF cells in three independent experiments. Distances from the edge of the cell sheet to the scratched line (solid line) were measured at five individual points. Data are presented as mean \pm standard deviation. Magnification, $x 100$; scale bar, $200 \mu \mathrm{m}$. " $\mathrm{P}<0.05$, compared with mock transfection control. siRNA, small interfering RNA. with Oct3/4 siRNA and after 2 days of culture, RNA was isolated and subjected to RT-qPCR to analyze the expression levels of Oct $3 / 4$ (Fig. 3A). It was observed that the expression levels of Oct $3 / 4$ were significantly downregulated following treatment with $200 \mathrm{nM}$ targeted-siRNA $(\mathrm{P}<0.05)$. The MTS assay demonstrated that cell proliferation was suppressed with Oct $3 / 4$ siRNA at 20 and $200 \mathrm{nM}$ compared with the mock control (both $\mathrm{P}<0.01$; Fig. 3B). Negative control siRNA also decreased cell proliferation compared with mock $(\mathrm{P}<0.05)$; however, Oct3/4 siRNA significantly decreased cell proliferation compared with the negative control siRNA at 20 and $200 \mathrm{nM}$ (both $\mathrm{P}<0.01)$.

Oct3/4 siRNA reduces the ability of HLF cells to migrate. To clarify the effect of Oct3/4 siRNA on cell motility, a scratch assay was performed. HLF cells were scratched with a sterile razor and transfected with Oct3/4 siRNA. After 2 days of culture, the transfected cells were imaged (Fig. 4A). The distance between the edge of the cell sheet and the scratched line was measured (Fig. 4B). It was observed that the distance significantly decreased in the layer of cells transfected with Oct $3 / 4$ siRNA, as compared with the mock transfection control $(\mathrm{P}<0.05)$.

\section{Discussion}

HCC cells are hypo-methylated in the promoter region of NANOG (14). Cancer stem cells are rare and a population may be enriched for cancer stem cells using serum-free media (15). In the present study, NANOG and Sox 2 were not expressed in any of the HCC cell lines. Therefore, it is hypothesized that the promoter region of NANOG may be methylated. However, it remains to be elucidated as to why Sox 2 is not expressed in HCC cells.

Oct3/4 is involved in pluripotency along with NANOG, Sox 2 and KLF4 (16). OCT3/4 is expressed in HCC cells (17), as well as being highly expressed in the cancer stem cells of HCC (18). Oct $3 / 4$ is upregulated by growth factors, including the insulin-like growth factor-1 via protein kinase B $(19,20)$. These previous studies suggest that Oct3/4 may affect the stemness of HCC cells, and also their phenotype. Higher expression levels of Oct $3 / 4$ are predictive of poor prognosis in patients with HCC (21). This previous study suggests that Oct $3 / 4$ has a role in carcinogenesis or cancer progression (21). In the present study, Oct3/4 siRNA significantly suppressed the proliferation and motility of HLF cells. These results indicate that Oct $3 / 4$ may be a potential therapeutic target for the treatment of HCC. This hypothesis was supported by Murakami et al (22), who demonstrated that Oct $3 / 4$ was a potentially effective target for differentiation therapy.

One limitation of the present study is that stemness markers were not analyzed in HLF cells following transfection with Oct $3 / 4$ siRNA. Therefore, the differentiation state of the cells was not determined. An investigation into the differentiation state of HCC cells transfected with Oct $3 / 4$ siRNA may facilitate further understanding. In conclusion, Oct $3 / 4$ is expressed in HLE, HLF and Hep3B cells, and Oct3/4 siRNA suppresses the proliferation and motility of HLF cells. 


\section{Acknowledgements}

This study was supported by a Grant-in-Aid for Scientific Research from the Japan Society for the Promotion of Science (grant no. 15K09032).

\section{References}

1. Cameron AM: Screening for viral hepatitis and hepatocellular cancer. Surg Clin North Am 95: 1013-1021, 2015.

2. Tomizawa M, Kondo F and Kondo Y: Growth patterns and interstitial invasion of small hepatocellular carcinoma. Pathol Int 45 352-358, 1995.

3. Tsujikawa H, Masugi Y, Yamazaki K, Itano O, Kitagawa Y and Sakamoto M: Immunohistochemical molecular analysis indicates hepatocellular carcinoma subgroups that reflect tumor aggressiveness. Hum Pathol 50: 24-33, 2016.

4. Tomizawa M, Garfield S, Factor V and Xanthopoulos KG: Hepatocytes deficient in CCAAT/enhancer binding protein alpha (C/EBP alpha) exhibit both hepatocyte and biliary epithelial cell character. Biochem Biophys Res Commun 249: 1-5, 1998.

5. Yamasaki H, Sada A, Iwata T, Niwa T, Tomizawa M, Xanthopoulos KG, Koike T and Shiojiri N: Suppression of $\mathrm{C} / \mathrm{EBP}$ alpha expression in periportal hepatoblasts may stimulate biliary cell differentiation through increased Hnf6 and Hnflb expression. Development 133: 4233-4243, 2006.

6. Tomizawa M, Wang YQ, Ebara M, Saisho H, Watanabe K, Nakagawara A and Tagawa M: Decreased expression of the CCAAT/enhancer binding protein alpha gene involved in hepatocyte proliferation in human hepatocellular carcinomas. Int $\mathrm{J}$ Mol Med 9: 597-600, 2002

7. Zaret KS and Carroll JS: Pioneer transcription factors: Establishing competence for gene expression. Genes Dev 25: 2227-2241, 2011.

8. Guye P, Ebrahimkhani MR, Kipniss N, Velazquez JJ, Schoenfeld E, Kiani S, Griffith LG and Weiss R: Genetically engineering self-organization of human pluripotent stem cells into a liver bud-like tissue using Gata6. Nat Commun 7: 10243 2016.

9. Takayama K, Inamura M, Kawabata K, Sugawara M, Kikuchi K, Higuchi M, Nagamoto Y, Watanabe H, Tashiro K, Sakurai F, et al: Generation of metabolically functioning hepatocytes from human pluripotent stem cells by FOXA2 and HNF1o transduction. J Hepatol 57: 628-636, 2012.

10. Takahashi K, Tanabe K, Ohnuki M, Narita M, Ichisaka T, Tomoda $\mathrm{K}$ and Yamanaka S: Induction of pluripotent stem cells from adult human fibroblasts by defined factors. Cell 131: 861-872, 2007
11. Tomizawa M, Shinozaki F, Motoyoshi Y, Sugiyama T, Yamamoto $\mathrm{S}$ and Ishige N: Niclosamide suppresses migration of hepatocellular carcinoma cells and downregulates matrix metalloproteinase-9 expression. Oncol Lett 10: 3515-3518, 2015.

12. Davies B and Fried M: The L19 ribosomal protein gene (RPL19) Gene organization, chromosomal mapping, and novel promoter region. Genomics 25: 372-380, 1995.

13. Tam S, Clavijo A, Engelhard EK and Thurmond MC: Fluorescence-based multiplex real-time RT-PCR arrays for the detection and serotype determination of foot-and-mouth disease virus. J Virol Methods 161: 183-191, 2009.

14. Wang XQ, Ng RK, Ming X, Zhang W, Chen L, Chu AC, Pang R, Lo CM, Tsao SW, Liu X, et al: Epigenetic regulation of pluripotent genes mediates stem cell features in human hepatocellular carcinoma and cancer cell lines. PLoS One 8: e72435, 2013.

15. Setoguchi T, Taga T and Kondo T: Cancer stem cells persist in many cancer cell lines. Cell Cycle 3: 414-415, 2004.

16. Nakai-Futatsugi Y and Niwa H: Transcription factor network in embryonic stem cells: Heterogeneity under the stringency. Biol Pharm Bull 36: 166-170, 2013.

17. Wu G, Wilson G, Zhou G, Hebbard L, George J and Qiao L: Oct4 is a reliable marker of liver tumor propagating cells in hepatocellular carcinoma. Discov Med 20: 219-229, 2015.

18. Zhu P, Wang Y, He L, Huang G, Du Y, Zhang G, Yan X, Xia P, Ye B, Wang S, et al: ZIC2-dependent OCT4 activation drives self-renewal of human liver cancer stem cells. J Clin Invest 125: 3795-3808, 2015.

19. Chang TS, Wu YC, Chi CC, Su WC, Chang PJ, Lee KF, Tung TH, Wang J, Liu JJ, Tung SY, et al: Activation of IL6/IGFIR confers poor prognosis of HBV-related hepatocellular carcinoma through induction of OCT4/NANOG expression. Clin Cancer Res 21: 201-210, 2015.

20. Zhao Y, Kong C, Chen X, Wang Z, Wan Z, Jia L, Liu Q, Wang Y, $\mathrm{Li} \mathrm{W}$, Cui J, et al: Repetitive exposure to low-dose X-irradiation attenuates testicular apoptosis in type 2 diabetic rats, likely via Akt-mediated Nrf2 activation. Mol Cell Endocrinol 422: 203-210, 2016.

21. Zhao RC, Zhou J, Chen KF, Gong J, Liu J, He JY, Guan P, Li B and Qin Y: The prognostic value of combination of CD90 and OCT4 for hepatocellular carcinoma after curative resection. Neoplasma 63: 288-298, 2016.

22. Murakami S, Ninomiya W, Sakamoto E, Shibata T, Akiyama $H$ and Tashiro F: SRY and OCT4 are required for the acquisition of cancer stem cell-like properties and are potential differentiation therapy targets. Stem Cells 33: 2652-2663, 2015. 